\title{
1 Unifying spatial and social network analysis 2 in disease ecology
}

3 Gregory F Albery ${ }^{1}$, Lucinda Kirkpatrick ${ }^{2}$, Josh A Firth ${ }^{3,4}$, Shweta

4 Bansal $^{1}$

5 1. Department of Biology, Georgetown University, Washington, DC, United States

6 2. EVECO, Institute of Biology, Universiteit Antwerpen, Antwerp, Belgium

7 3. Edward Grey Institute, Department of Zoology, University of Oxford, Oxford, UK

8 4. Merton College, Oxford University, Oxford

9 Abstract

10 1. Social network analysis has achieved remarkable popularity in disease ecology, and is 11 sometimes carried out without investigating spatial heterogeneity. Many investigations into 12 sociality and disease may nevertheless be subject to cryptic spatial variation, so ignoring 13 spatial processes can limit inference regarding disease dynamics.

142 . Disease analyses can gain breadth, power, and reliability from incorporating both spatial and 15 social behavioural data. However, the tools for collecting and analysing these data 16 simultaneously can be complex and unintuitive, and it is often unclear when spatial variation 17 must be accounted for. These difficulties contribute to the scarcity of simultaneous spatial18 social network analyses in disease ecology thus far.

19 3. Here, we detail scenarios in disease ecology that benefit from spatial-social analysis. We describe procedures for simultaneous collection of both spatial and social data, and we outline statistical approaches that can control for and estimate spatial-social covariance in disease ecology analyses.

4. We hope disease researchers will expand social network analyses to more often include spatial components and questions. These measures will increase the scope of such analyses, allowing more accurate model estimates, better inference of transmission modes, 
susceptibility effects and contact scaling patterns, and ultimately more effective disease interventions.

\section{Introduction}

29 Spatial structuring is ubiquitous, and can influence all conceivable intrinsic and extrinsic factors 30 in disease ecology. As such, not accounting for space can weaken analyses (Pawley \& McArdle,

31 2018; Pullan, Sturrock, Soares Magalhaes, Clements, \& Brooker, 2012; Tobler, 1970). Although

32 spatial effects can potentially touch any process, social interactions may be particularly

33 vulnerable (Adams, Faust, \& Lovasi, 2012). Consequently, the relationship between ecology-

34 driven spatial structure and fine-scale social interactions has shaped the study of animal societies

35 for decades. The recognition that social systems are structured by the surrounding environment

36 rather than comprising random arrangements of independent individuals (Crook, 1964; Crook \&

37 Gartlan, 1966) was followed by foundational theory stating that ecological factors influence the

38 spatial distribution of individuals within populations, which in turn determines which individuals

39 interact (Clutton-Brock, 1974; Crook, 1970). Recently, the relationship between spatial

40 structuring and sociality has been addressed in the context of animal social networks (Krause,

41 James, Franks, \& Croft, 2015; Webber \& Vander Wal, 2019); although relatively well-

42 understood in the context of animal behaviour itself, the role of the environment and spatial

43 behaviour requires addressing more frequently in studies that investigate social correlates of

44 disease.

Spatial behaviour can influence social network analyses of wildlife disease through a few

47 principal mechanisms, which we discuss in Section 3. Fundamentally, it is important to remember that the social environment exists within space, so whom an individual spatially overlaps with defines who they can socially interact with (Whitehead, 2008). Consequently, the spatial and social networks often reinforce, or represent, one another, and their correlation may

51 require controlling for (3A), or can be leveraged for operational purposes (3B). Additionally,

52 social network traits can covary with many spatial processes. For example, many pathogens

53 transmit through the environment, so -in this cases- spatial behaviours define relevant 'contact

54 events' rather than social ones, or social contact events may be spatially structured (3C). 
Likewise, host immunity and susceptibility are determined by environmentally varying gradients

56 in climate and resource availability, which could counteract or artificiate social effects (3D).

57 Finally, a common question in disease ecology concerns the scaling of contact events with

58 population density, known as "density dependence"; in section 3E, we pose this question as a

59 spatial-social question, and outline how spatial-social methods could be used to address the

60 problem in future analyses.

62 Ultimately, we summarise how spatial and social behaviour can influence infection (Figure 1),

63 and present a conceptual framework of how to analyse them simultaneously (Figure 2). We start

64 by defining both behaviours (Section 1) and discussing why their unified analysis is relatively

65 rare in disease ecology (Section 2), and then outlining reasons to analyse both where possible

66 (Section 3, described above). To help researchers with tackling spatial-social questions, we then

67 outline methods by which space and sociality can be delineated at the data collection level

68 (Section 4; Box 1), particularly focussing on methods that involve approximating social

69 behaviour with parameterisations of spatial behaviours. We then give case studies for

70 considering spatial-social systems (Box 2), and approaches for simultaneous spatial-social

71 analysis (Section 5). Specifically, we discuss the distinction between controlling for space or

72 sociality, and alternative spatial analysis methods that explicitly quantify both spatial and social

73 processes. Finally, we outline important emerging frontiers and model systems in which the

74 ongoing study of spatial and social behaviour is increasingly important and revealing (Section 6).

75 In doing so, we provide an optimistic guide to conducting spatial-social analyses in the future,

76 encouraging new and exciting investigations in the field of network disease ecology.

77 1. How to define spatial and social behaviour

78 We define "spatial behaviour" (or "space") as any representation of an individual's context

79 within its surrounding environment (Pullan et al., 2012). This may comprise point locations in

80 space (Albery, Becker, Kenyon, Nussey, \& Pemberton, 2019), movement trajectories (Mourier,

81 Lédée, \& Jacoby, 2019), space use distributions (Stopher et al., 2012), or a description of

82 surrounding environmental variables (Saito \& Sonoda, 2017). Note that in the latter case,

83 environmental variables are counted as a spatial measure, but by definition they must be taken 
84 relative to an organism's spatial context. For example, if a researcher may be interested in the

85 role of environmental temperature in driving between-individual variation in parasitism, they must first decide whether to use temperature readings from near each animal's point locations, or averaged across each individual's home range. Meanwhile, we define "social behaviour" broadly as any social association between individuals (Croft, James, \& Krause, 2008). Dyadic social connections can be inferred from all nature of social associations, ranging from direct interactions involving physical contacts (e.g. grooming, mating, fighting), to implied associations such as co-occurrence in fission-fusion social groupings (e.g. pods of marine mammals, foraging flocks of birds) known as the gambit-of-the-group approach (Franks et al 2010). Crucially, just as incorporating multiple social behaviours and network metrics can help with hypothesis testing

94 (Sosa, Sueur, \& Puga-Gonzalez, 2020), simultaneously investigating multiple spatial behaviours can be extremely helpful in revealing the underlying mechanisms in a wildlife system (Albery,

96 Morris, et al., 2020).

\section{Why is space understudied in disease ecology social} 98 network analyses?

99 Network disease ecology suffers from a lack of methodological workflows and tools for dealing with spatial-social confounding, contributing to our lack of understanding of the relative

101 importance of spatial and social behaviours. Both are hard to investigate, and studies are rarely

102 designed with both in mind, so assessing them simultaneously can be difficult. Many studies 103 experience operational limitations in detecting spatial variation: for example, ecoimmunological

104 sampling regimes often attempt to minimise spatial variation rather than investigating it directly, 105 rarely use spatial analysis methods, and generally have few spatial replicates (Becker et al., 106 2020), which may reduce their power to detect spatial variation (Becker et al., 2019). Fitting 107 spatial models can require specialist knowledge which may contribute to the widespread 108 impression that space is more difficult to analyse than social connectivity; however, this is no 109 truer of spatial analysis than it is of social network analysis. Additionally, the field of social 110 network ecology has historically employed network permutations that analytically control for the 111 effect of spatial behaviour to ensure that spatial confounding is not responsible for an observed 112 effect (Farine, 2013). On the contrary, rather than perceiving space simply as something "to 
113 control for", it is far more productive to treat space as an exciting and useful component of a

114 system's biology that is worthy of explicitly quantifying in its own right (Albery et al., 2019;

115 Pawley \& McArdle, 2018).

116

117 Limitations likewise apply to the collection of spatially explicit social data. Because social

118 behaviour can be hard to observe or infer, some social network analyses use spatiotemporal

119 proximity to approximate social interactions (Farine, 2015; Gilbertson, White, \& Craft, 2020;

120 Wanelik, 2019). This method is used frequently enough that tools have been developed to

121 calculate social associations directly from spatiotemporal data (e.g. the spatsoc R package;

122 Robitaille, Webber, \& Vander Wal, 2019). This heuristic may introduce spatial-social

123 confounding in some systems, and it is not necessarily true that social contacts will correlate

124 perfectly (or even that well) with space, so using one to approximate the other may or may not be

125 valid (Castles et al., 2014; Gilbertson et al., 2020; but see Farine, 2015). The definitions for these

126 behaviours are especially important in disease ecology because the field revolves around

127 pathogens that are spread by contact events arising from them. For example, if a study of directly

128 transmitted pathogens assumes that spatial collocations represent social contacts when in fact

129 they do not, the study may be fundamentally unable to draw accurate conclusions about

130 transmission (Section 3C). It is therefore vital that spatial and social behaviours be defined

131 correctly and delineated from each other for disease network analyses to function as intended

132 (Leu, Sah, Krzyszczyk, Jacoby, \& Mann, 2020; Manlove et al., 2018; Richardson \&

133 Gorochowski, 2015; Sih, Spiegel, Godfrey, Leu, \& Bull, 2018).

135 Encouragingly, there has been considerable recent progress identifying the importance of

136 separating space and sociality in network studies of animal behaviour (Mourier \& Jacoby, 2019;

137 Silk, Finn, Porter, \& Pinter-Wollman, 2018; Webber \& Vander Wal, 2018; see Case Studies).

138 This push is likewise true in disease ecology, as demonstrated by increasing calls for

139 incorporation of spatial effects in network analyses, particularly where indirectly transmitted

140 pathogens are concerned (Sih et al., 2018; Silk et al., 2019; White, Forester, \& Craft, 2017).

141 Moreover, there is increasing conceptual and methodological overlap among the fields of

142 movement ecology, network science, and disease ecology (Dougherty, Seidel, Carlson, Spiegel, 
143 \& Getz, 2018; Jacoby \& Freeman, 2016). As such, the time is ripe for increased synthesis of 144 spatial and social network methodology in disease ecology studies where possible.

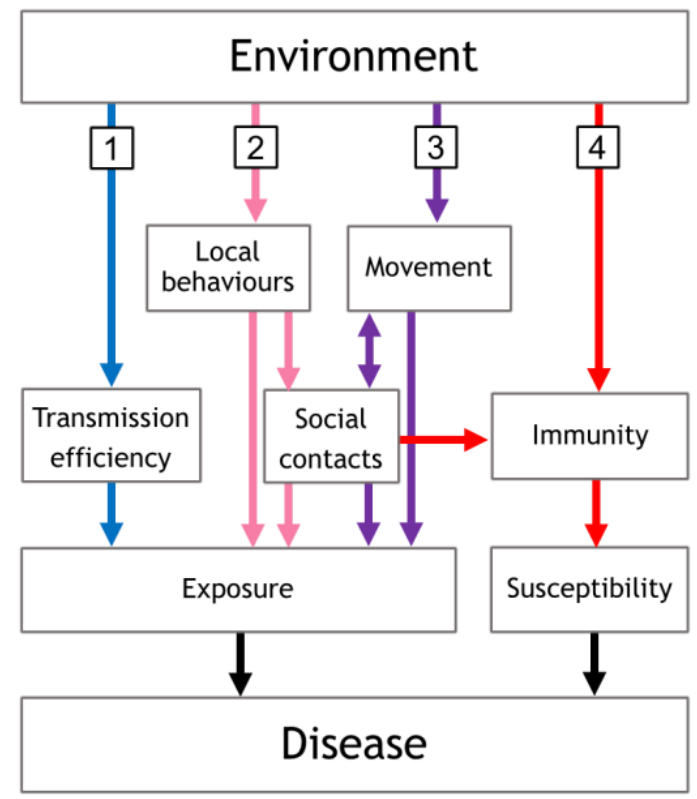

Figure 1: Principal causal pathways among the environment, spatial behaviour, sociality, and disease. 1 (blue lines): Environmental variation in climatic factors affects the transmission efficiency of indirectly transmitted parasites. 2 (pink lines): The environment drives spatial variation in specific social behaviours such as fighting and mating, driving spatial variation in

153 structure and resource distribution determines movement patterns, which themselves determine 154 the social network. Movement patterns determine exposure to indirectly transmitted parasites.

155 The social network determines exposure to directly transmitted parasites, as well as determining 156 susceptibility through changes in resource acquisition and stress. Spatial behaviour and social 157 behaviour can interact. 4 (red lines): The distribution of resources in the environment affects allocation to immunity, creating spatial variation in susceptibility to parasites. 
162 operational benefits. Below we consider several of these advantages. Fundamentally, we argue 163 that spatial-social analysis is important because it is challenging to predict where spatial and 164 social behaviours interact, and potentially compete, in influencing disease dynamics. Although 165 spatial-social correlations are common (e.g. Firth \& Sheldon, 2016; Mourier \& Jacoby, 2019;

166 O’Brien, Webber, \& Vander Wal, 2018), these relationships vary considerably across systems, 167 and can be context-dependent (e.g. O'Brien et al., 2018). Unfortunately, little consensus is 168 available on which systems and environments are most likely to exhibit spatial-social 169 correlations due to the rarity of cross-system synthesis. Although recent studies have integrated social networks across a range of animals to make strong comparative conclusions (Sah, Mann,

$171 \&$ Bansal, 2018), spatial-social relationships have evaded the same scrutiny. Additionally, fine-

172 scale spatial analyses of wildlife disease are themselves rare and similarly lacking in cross-

173 system comparisons. As such, it is difficult to predict a priori which systems and sampling

174 regimes will exhibit the most spatial-social confounding. This uncertainty alone is a strong

175 reason to incorporate spatial analyses into social network studies of wildlife disease.

177 There likely exist certain systems for which spatial-social analysis is unnecessary, and social

178 network analysis alone is sufficient. However, although it is tempting, we opt not to speculate on 179 these systems for the following reasons: first, the lack of cross-system syntheses means there is 180 currently little empirical evidence which would allow actual assessment, so most such

181 recommendations would be mostly conjecture. Second, the numerous advantages cover so many 182 factors that there are few systems that would not benefit in at least one way by conducting a 183 spatial-social analysis (even if this demonstrated the relative unimportance of space). In the 184 future, greater application of spatial (or spatial-social) analyses of wildlife disease, and 185 increasing application of simulations aimed to answer these questions (e.g. Gilbertson et al., 186 2020), may help to clarify these issues for a wider range of studies, providing more prescriptive 187 guidelines.

\section{A. Controlling for habitat selection and spatial-social feedbacks}

189 The landscape defines the distribution of resources and potential movement paths, which shapes 190 the structure of the social network through habitat selection (Figure 1; (Albery, Morris, et al., 191 2020; He, Maldonado-chaparro, \& Farine, 2019; Webber \& Vander Wal, 2018). Reciprocally, 
192 the social environment forms an important component of survival, competition, and dispersal in

193 a heterogeneous environment (Armansin et al., 2019). As such, at fine scales, animals may make

194 space use decisions based on their associates', weighed against environmental cues (Firth \&

195 Sheldon, 2016; Peignier et al., 2019). Given this strong mutual causality, it can be difficult to say

196 whether any behaviour represents solely spatial or social processes.

198 Empirical attempts to delineate spatial and social behaviour are complicated when considering

199 interactions with disease. Both spatial and social behaviour determine an individual's exposure

200 and susceptibility to infection, and yet behaviour, being highly plastic, can also change in

201 response to infection (Ezenwa, Archie, et al., 2016). For example, sickness behaviours often

202 induce sluggishness and a reduction in social activity (Lopes, 2014; Lopes, Block, \& König,

203 2016). It is often mechanistically unclear whether this reduced sociality is an active process,

204 serving e.g. to avoid infecting close relatives or conspecifics, or whether energy-saving

205 reductions in movement merely result in a reduction in sociality by extension (Lopes, Block,

206 Pontiggia, Lindholm, \& König, 2018). In addition, parasites commonly affect animals’

207 movement decisions, e.g. through parasite avoidance behaviours, so the spatial distribution of

208 diseases in the environment can determine animals' distributions through a "landscape of

209 disgust" in the same way that predators define a "landscape of fear" (Albery, Newman, et al.,

210 2020; Weinstein, Buck, \& Young, 2018). This phenomenon could produce complex covarying

211 patterns: for example, if habitat selection and life history traits covary with immunity and

212 parasite avoidance (Hutchings, Judge, Gordon, Athanasiadou, \& Kyriazakis, 2006), the emergent

213 social network could demonstrate artefactual clustering in susceptibility.

215 Nevertheless, extricating the roles of spatial and social behaviour in driving disease is not a futile

216 endeavour. Behaviours can be classified on a continuum from "more spatial" (e.g. map locations)

217 to "more social" (e.g. partner choice), and examining and comparing their influence on parasite

218 burden will similarly reveal whether the drivers of parasitism are more likely to be spatial or

219 social. Although some study systems may be poorly suited to spatial-social analysis due to

220 observation difficulties, in most cases fitting both spatial and social behaviours in a model and

221 comparing their effects will likely strengthen inference beyond study designs incorporating only

222 one of the two (see Analysis section). 


\section{B. Simplifying measurement approaches}

224 In some circumstances, well-understood spatial-social confounding may be leveraged for 225 operational benefits: for example, streamlining data collection and disease surveillance in wild 226 animal populations with sparse data. Collecting copious GPS data is easier than ever (Kays,

227 Crofoot, Jetz, \& Wikelski, 2015) and can be carried out remotely, while social phenomena can

228 be much harder to observe directly (see Box 1). Where spatial data are easier to collect than 229 social interactions, verifying that the two correlate may allow the use of spatial data to

230 approximate social contacts, or social networks and contact events are commonly approximated 231 using parameterised movement data (see below, Box 2 and Section 4). For example, a study of

232 African domestic dog populations used GPS tracking and proximity loggers to demonstrate that 233 individual home range size correlated well with network centrality, which in turn influenced

234 individual propensity to spark simulated rabies epidemics (Wilson-Aggarwal et al., 2019).

235 Similar logic could apply to any system in which ranging behaviour covaries predictably with 236 sociality; however, strong spatial-social correlations are not ubiquitous. Given this uncertainty, 237 we stress that this approach should only be taken cautiously and when accompanied by rigorous 238 validation procedures. In any case, empirical measures of sociality and spatial behaviour will 239 often be imperfect proxies for the interactions that researchers hope to quantify (Farine, 2015).

240 Attempting to incorporate both space and sociality in concert may buffer for this necessity.

\section{C. Identifying pathogen transmission mode}

242 While recent work has considered how the spread of information, or behaviours, may depend on 243 the fine-scale transmission mode between individuals (Firth 2020 TREE), similar considerations 244 also apply to parasite transmission. Indeed, unknown parasite transmission mode is a common 245 reason for conducting spatial-social analyses. Contact events can arise from a variety of 246 spatial/social processes, so the relative importance of spatial and social behaviour depends 247 heavily on the pathogen's transmission mode. Therefore, where transmission mechanisms are 248 unknown, incorporating both spatial and social behaviour helps identify the pathogen's

249 transmission mode, because the behaviour that most closely approximates contact events will 250 best describe variation in infection (Craft, 2015; White et al., 2017). Intuitively, environmental 251 variables will only weakly influence individuals' exposure to directly transmitted pathogens, and 
252 transmission probability will most accurately be represented by social proximity. As such, if

253 space is found to be unimportant relative to sociality, researchers can conclude that direct

254 transmission is likely. For example, in sleepy lizards (Tiliqua rugosa), social proximity was a

255 better predictor of Salmonella transmission than was spatial proximity, indicating a relatively

256 direct mechanism (Bull, Godfrey, \& Gordon, 2012). Conversely, simultaneous use of proximity

257 loggers and GPS tracking revealed that badgers and cattle rarely contact each other directly

258 (despite substantial range overlap), indicating that bovine tuberculosis (Mycobacterium bovis) is

259 likely transmitted through the environment (Woodroffe, Donnelly, Ham, Jackson, \& Moyes,

260 2016). An important distinction should be made between pathogens that are transmitted through

261 specific social interactions (e.g., sexually transmitted infections) and those that merely require

262 spatiotemporal coincidence (e.g., aerosol-transmitted viruses). It is possible that both spatial and

263 social behaviours will have detectable, non-interchangeable effects on transmission patterns for

264 the latter group of pathogens, so that both behaviours are needed to gain a full picture of disease

265 dynamics.

267 Ignoring transmission mode when examining correlates of spatial/social behaviour can produce a 268 confusing picture of a system's ecology. For example, a study in Japanese macaques (Macaca 269 fuscata) found that centrality in the grooming network was positively correlated with infection 270 with indirectly transmitted nematodes, which seems mechanistically unlikely (MacIntosh et al., 271 2012). It is possible that the nematodes' transmission mode is poorly understood, exhibiting a 272 more direct, social component, but it is also possible that the grooming network was spatially 273 structured, so that social network centrality reflected environmental processes rather than 274 sociality itself (MacIntosh et al., 2012). Importantly, because the environment may determine 275 aspects of individual behaviour decisions, some geographic areas may be hotspots for contact 276 events (Albery, Morris, et al., 2020) or for certain risky behaviours, even where the pathogen is 277 directly transmitted. For example, if certain areas lend themselves to fighting or mating grounds 278 for Tasmanian devils (Sarcophilus harrisii), this would create enduring spatial variation in the 279 prevalence of Tasmanian devil facial tumour disease despite strictly direct transmission (Figure 280 1; Hamede, Bashford, McCallum, \& Jones, 2009). Therefore, known transmission mode is not 281 sufficient to predict whether or not space is worth investigating in a given host-parasite system, 282 and researchers will benefit from measuring both. 
284 Social network analyses commonly focus on the role of social contact events in driving parasite 285 exposure. However, it is important to bear in mind that parasite burden is also a function of 286 susceptibility, that the spatial and social environments can impact host immunity directly, and 287 that these effects may not align (Albery et al., 2019; Becker et al., 2018, 2019). As such, space 288 and sociality should be quantified simultaneously if there is any expectation that they will affect

289 both susceptibility and exposure. Resource supplementation provides an ideal example:

290 increased food should provide more resources for allocation to immunity, reducing

291 susceptibility, yet supplementation commonly leads to aggregation on feeding sites, increasing 292 exposure rates as a result (Becker, Streicker, \& Altizer, 2015). Consequently, supplementation 293 could either increase or decrease parasitism, or neither, depending on the balance of these 294 processes. Interestingly, the social environment can also alter susceptibility through stress295 induced immunosuppression, potentially counteracting environmental effects on susceptibility or 296 transmission (Ezenwa, Ghai, McKay, \& Williams, 2016; Hawley, Etienne, Ezenwa, \& Jolles, 297 2011). Examining both spatial and social behaviour simultaneously may help to extricate 298 sociality-driven changes in susceptibility when examining environmentally transmitted 299 pathogens. One of the foremost advantages of measuring immunity in conjunction with 300 parasitism lies in distinguishing susceptibility- and exposure-driven processes (Bradley \& 301 Jackson, 2008). We suggest that studying immunity alongside space, sociality, and parasitism 302 will similarly bolster the strength of inference in determining transmission mechanisms while 303 accounting for susceptibility effects in network disease ecology.

\section{E. Quantifying density dependence}

305 Epidemiological models often make fundamental assumptions about the scaling between 306 population density, contact events, and disease (i.e., "density-dependence"), and the validity of 307 these assumptions can profoundly alter models' ability to predict disease dynamics (Antonovics, 308 2017; Hopkins, Fleming-Davies, Belden, \& Wojdak, 2020). This question is fundamentally a 309 spatial-social one: how do interactions increase when you add more individuals to the same 310 space? For example, adding more individuals in a given space will generally result in an in-step 311 increase in aerosol inhalation, producing increased contact events for droplet-transmitted 
312 pathogens; however, such increased host density will not necessarily result in a proportional

313 increase in copulation events, so sexually transmitted infections (STI's) are unlikely to scale in

314 this way. As such, STI's are generally considered "frequency-dependent". In reality, all

315 pathogens exist somewhere on a continuum between the two, and identifying where they are

316 placed is an important research priority (Hopkins et al., 2020).

318 Despite its relative rarity in disease ecology, spatial-social analysis could be incredibly revealing

319 when it comes to empirically identifying pathogens' density dependence and the scaling of

320 contact events. In the absence of disease data, spatial-social analyses could reveal whether

321 increased population density results in a greater frequency of interactions or associations, and

322 this information could be incorporated into epidemiological models. Alternatively, researchers

323 could incorporate both spatial population density and social network metrics at the individual

324 level to identify which best describes disease burden, informing how density and interaction

325 frequency compare (e.g. (Albery, Newman, et al., 2020). Unfortunately, as yet most

326 investigations into density-dependence are conducted post hoc, and there is no framework for $a$

327 priori prediction of density dynamics in novel host-pathogen systems. This fact may hamstring

328 efforts to develop epidemiological models and interventions, particularly in the case of novel

329 pathogen emergence, and increasing use of spatial-social approaches could address this gap. 


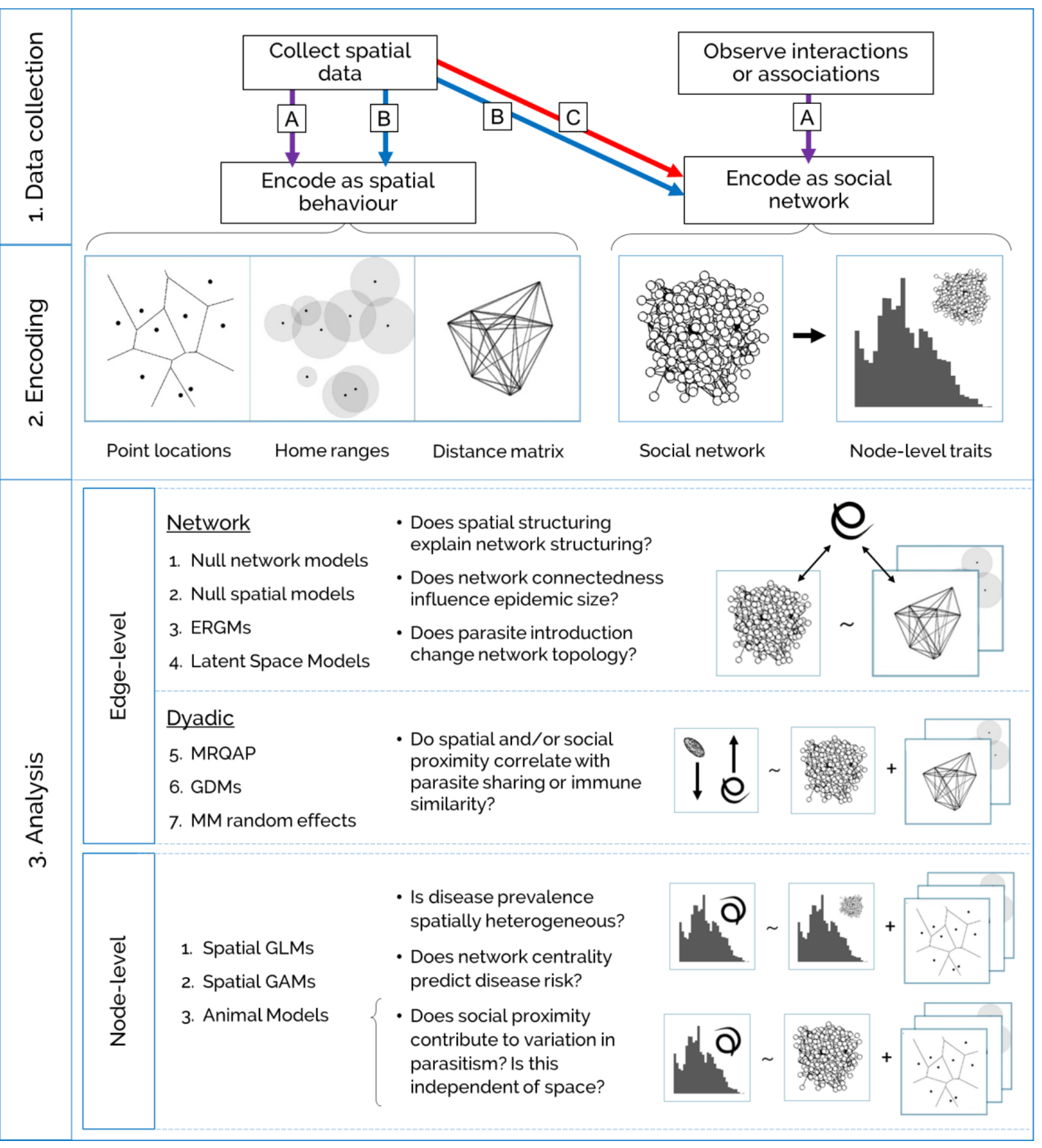

Figure 2: Proposed workflow for collecting, encoding, and analysing spatial data alongside social

333 network data. Section 1: Data collection. Purple, blue, and red arrows represent study design options A,

334 B, and C respectively; see "Collecting spatial behaviour with social data". Section 2: Encoding methods.

335 Ways to encode spatial behaviour, as either a node-level or dyadic trait. These include: Centroids (point 336 locations) taken from $\mathrm{N}>=1$ observations of individuals. Individual territories have been assigned using 337 Voronoi tessellation (black lines). Point locations can also be used to create home ranges or distance 
matrices, or fitted as an autocorrelation function in a statistical model examining node-level traits. Home ranges (grey circles) can be calculated from multiple sightings or derived from movement patterns or kernels, and then coded as a square similarity matrix of range overlaps, to be used in edge-level analyses or as variance components in node-level animal models. Pairwise distances (lines) can be taken between point locations and coded as a square similarity matrix, to be used similarly to home range overlap. Line thickness and opacity are inversely proportional to distance. Section 3: Analysis methods. Statistical approaches to analyse spatial-social disease processes and some example questions that each can answer. GLM = Generalised Linear Model; GAM = Generalised Additive Model; Animal Models $=$ a model with a dyadic variance component included; ERGMs = Exponential Randomised Graph Models; MRQAP = Multiple Regression Quadratic Assignment Procedure; GDM = Generalised Dissimilarity Models; MM random effects $=$ Multi-Membership random effects.

\section{Box 1: Methods for collecting spatial and social data simultaneously}

Spatial data can take Lagrangian or Euclidean forms, each representing a different way of perceiving movement across the landscape (Nathan et al., 2008; Smouse et al., 2010). Lagrangian data collection (GPS, censusing, and motion tracking) involves the researcher conceptually moving through space, following individuals and summarising their movements. Euclidean data collection (trapping regimes and proximity loggers) uses static sampling locations which collect data on animals moving around them. Lagrangian data are richer and offer greater opportunities for parameterisation; however, Euclidean data collection locations are generally placed by the researcher, so they can be economically distributed in space to cover large areas with minimal effort and/or to accompany visits to locations of biological relevance or experimental manipulation sites (e.g. Firth \& Sheldon, 2015). The optimal choice of methods will depend on operational constraints imposed by the study system of interest, e.g. with regards to the size of the animal, the area over which it ranges, and the pathogen and biological process of interest. Here, we outline several methods of spatial-social data collection, including a brief summary of each approach, how they can be used to quantify spatial behaviour and social

368 GPS: animals are marked and tracked over relatively large distances using satellites. 
369 Spatial: summarise individuals' movements across the landscape.

370 Social: parameterise activity patterns to identify groups or interactions.

371 Examples: cattle (Woodroffe et al., 2016); cheetahs (Broekhuis, Madsen, Keiwua, \&

372 Macdonald, 2019); feral dogs (Wilson-Aggarwal et al., 2019).

374 Motion tracking cameras: when the study organism is in a contained space, a large proportion 375 of the population is observed using motion-tracking technology.

376 Spatial/Social: same as GPS, above.

377 Examples: carpenter ants (Modlmeier et al., 2019); Lasius niger ants (Stroeymeyt et al., 2018).

379 Census routes: researchers follow a predetermined or random route around a study area and 380 record individual animals' behaviour.

381 Spatial: record locations of individuals or groups.

382 Social: record group memberships or interactions between individuals.

383 Examples: dolphins (Frère et al., 2010; Lusseau et al., 2006); red deer (Stopher et al., 2012).

Spatial proximity loggers: loggers are placed on individuals and in specific environmental 386 locations to identify contact events.

387 Spatial: use individuals' environmental contact locations to create models of spatial behaviour.

388 Social: use individuals' contact events to create proximity/interaction/social networks.

389 Examples: Mastomys rodents (Berkvens, Olivares, Mercelis, Kirkpatrick, \& Weyn, 2019); great

390 tits (Firth \& Sheldon, 2016); European badgers (Woodroffe et al., 2016); reef sharks (Jacoby,

391 Papastamatiou, \& Freeman, 2016).

393 Trapping locations: animals are captured for sampling or camera traps used to identify 394 individuals.

395 Spatial: record individuals' trapping locations, summarising across repeated trapping events.

396 Social: record individuals trapped in the same group or within a given spatiotemporal window.

397 Examples: vole trapping (Davis et al., 2015; Wanelik, 2019); hyena camera traps (Stratford, 398 Stratford, \& Périquet, 2019). 
400 Box 2: Frameworks for delineating and analysing spatial and social

401 behaviour

402 Given the well-understood nature of spatial-social behaviours, there are a great many studies that 403 examine their covariance, and several frameworks have been developed to help untangling and 404 analysing them. Here, we describe some case studies that provide such frameworks to guide 405 researchers carrying out spatial-social analyses of disease processes.

\section{A tripartite network scaffolding for spatiotemporal contact patterns}

Manlove et al. (2018) developed a tripartite network which allows characterisation of contact events using three classes of node: space, time, and individuals. Using multiple real-world examples, they demonstrated that this network can be collapsed to form spatial and social networks that are commonly employed in disease ecology. Moreover, the tripartite network was valid for multiple different social systems. Although general and highly flexible, the approach

414 the derived contacts are most applicable for directly transmitted parasites (Manlove et al., 2018).

415 An important expansion of the framework will be to incorporate spatiotemporal variation and lag 416 times (Richardson \& Gorochowski, 2015; see Timescale Section).

418 Connecting habitat selection and socio-spatial behaviour with eco-evolutionary 419 consequences.

420 Webber \& Vander Wal (2018) outline a comprehensive eco-evolutionary framework for spatial421 social behavioural integration. Specifically, they link individual-level habitat selection 422 behaviours with spatial movements, and then outline how this spatial behaviour results in the 423 development of social networks. They discuss how the resulting framework can be used to 424 examine fitness consequences and ecological dynamics, using animal models, among other 425 approaches (see analysis section). Their incorporation of spatial-social behaviours into 426 quantitative genetic models offers a useful framework for identifying individual-level fitness 427 consequences (and their genetic determinants) while accounting for environmental confounders 428 and density dependence. Their paper offers an interesting scaffold for the investigation of 
429 divergent effects of density-driven susceptibility and exposure effects, and the implied costs and 430 benefits of sociality for disease (Ezenwa, Ghai, et al., 2016).

\section{Networks of networks in reef shark movement ecology}

433 Mourier \& Jacoby (2019) used reef sharks as a case study to construct a movement ecology-

434 based framework for spatial-social analysis. In this approach, individuals' movement trajectories 435 are represented as networks, where each node of the network is a Euclidean sampling location, 436 and edges are represented by the individual's movements between these locations. The adjacency 437 matrices from these networks are then nested in a super-adjacency matrix for further analysis, 438 forming a "network of networks". This framework benefits from the fine data resolution it 439 allows, avoiding collapsing individuals' movements into summary statistics such as point 440 locations or space use distributions (Figure 2, Section 2). The authors used this approach to 441 demonstrate high covariance between sharks' spatial and social centrality (Mourier et al., 2019).

442 Like the tripartite model above, this framework is designed for Euclidean sampling locations 443 fixed in space, and has not yet been adapted for Lagrangian data; as such, Lagrangian systems 444 may need to (artificially) discretise their spatial data to take a similar approach.

446 Competing multiple spatial and social metrics to deconstruct density dependence in 447 a group-living carnivore

448 (Albery, Newman, et al., 2020) examined parasite burdens in European badgers (Meles meles) to 449 investigate socio-spatial drivers. They fitted a series of models with either social metrics (group 450 size and co-trapping networks) or spatial population density, revealing that areas with high 451 population density unexpectedly had lower parasite burdens. Because purely social metrics 452 meanwhile had no detectable effects, cooperative grooming was unlikely to be the cause of the 453 negative density dependence. A series of subsequent analyses revealed that spatial avoidance of 454 parasite transmission was most likely responsible. 


\section{4. Collecting spatial behaviour with social data}

457 If spatial-social analysis is to be carried out, researchers must first collect both data types. Three

458 main study design options can incorporate both spatial and social data collection (Figure 2,

459 Section 1): A) collect both spatial and social data separately, and encode them as different

460 networks; B) collect only spatial data, using spatiotemporal parameters to estimate contact

461 events; or C) collect only spatial data, using these to approximate social contacts without further

462 parameterising - e.g., where spatial proximity is expected to directly represent social proximity.

463 Although the latter is occasionally the only available option for quantifying social behaviour in a

464 given system, we discourage this method for the reasons outlined above.

465 What spatial measures are available?

466 Data collection methods for social networks can take many forms, and have been well-reviewed 467 elsewhere (Craft, 2015; Krause et al., 2015; White et al., 2017). Many such methods do not 468 necessarily involve an explicit spatial component, yet they can often be extended to do so with

469 little difficulty. In Box 1, we provide a non-exhaustive list of methods that can be used to collect 470 both spatial and social behaviours simultaneously. Once data have been collected, there are 471 several possible options for encoding spatial behaviour for use in network analyses (Figure 2, 472 Section 2). It is important to consider whether a given spatial measure represents location effects 473 (i.e., where an individual is on a variable landscape) or space sharing effects (i.e., the similarity 474 or proportional overlap of two individuals' spatial environments; Albery, Morris, et al., 2020; 475 Pullan et al., 2012). The two may correlate - e.g., individuals living closer together will share 476 more of their home ranges - but these different types of spatial behaviour can operate differently, 477 potentially offering different insights, and may have additive benefits for inference when 478 considered simultaneously (Albery, Morris, et al., 2020). The relative advantages of the spatial 479 measures used may depend on the system itself: for example, home range overlap will be 480 uninformative for parasitism when species are territorial or at such low density that their home 481 ranges rarely overlap. Pairwise distances and home range overlap matrices can be conceptualised 482 as a spatial network, if this helps with statistical analysis (Figure 2, Section 2; see analysis 483 section; Mourier et al., 2019). 


\section{Pairing and delineating spatial and social behaviour}

485 To carry out spatial-social analysis, researchers will need to distinguish social behaviours from 486 spatial activity/occurrence either methodologically or statistically (Figure 2; Box 1).

487 Methodologically distinguishing the two involves either combining two data collection methods, 488 each designed to pick up different behaviours, or using multiple types of observations collected 489 by researchers (Figure 2, option A). For example, GPS can provide good wide-resolution spatial 490 data while proximity loggers are used simultaneously to build networks of close-range 491 interactions among individuals (Ossi et al., 2016). Alternatively, researchers conducting

492 behavioural censuses can collect social data by identifying associating or interacting individuals, 493 while also recording spatial locations. The associations/interactions produce a social association 494 network, while the point locations or derived home range estimates provide spatial information.

Distinguishing spatial and social behaviours statistically (post-data collection) involves

497 parameterising high-resolution (Lagrangian) behavioural data (Figure 2, option B). For example, 498 GPS-tracking wide-ranging territorial species such as cheetahs (Acinonyx jubatus) provides 499 movement data from which contact events can be reasonably inferred purely because individuals 500 rarely come into close proximity of each other (Broekhuis et al., 2019). Meanwhile, the home 501 ranges of the individuals can be independently derived from GPS patterns, and controlled for 502 separately (Seidel, Dougherty, Carlson, \& Getz, 2018). Alternatively, study organisms such as 503 ants can be recorded to track the movements of each individual, with contact events identified 504 within this spatial behaviour (e.g. Stroeymeyt et al., 2018). Both of these methods involve 505 selecting defensible criteria for contact events, based on stereotyped behaviours, approach 506 patterns/trajectories (Schlägel et al., 2019), or spatiotemporal proximity (Robitaille et al., 2019). 507 Sophisticated algorithms such as Gaussian mixture models can be used to infer grouping events 508 (Firth et al., 2017; Psorakis et al., 2015) or interactions (Jacoby et al., 2016), avoiding the 509 necessity of defining arbitrary criteria. Encouragingly, even complex, asymmetrical interactions 510 can be identified using only parameterised movement patterns (Jacoby et al., 2016; Schlägel et

511 al., 2019), potentially helping disease ecology researchers to infer specific contact events 512 contributing to transmission. 
514 Many studies have examined spatial-social behaviours and their covariance without necessarily

515 tying them to disease ecology; this includes study systems such as great tits (Firth \& Sheldon,

516 2016); elk (O’Brien et al., 2018); sharks (Mourier et al., 2019); and many more. Because of the

517 longstanding interest in their simultaneous analysis, several helpful frameworks have been

518 developed; we describe some in Box 2.

\section{Spatial-social analysis methods in disease ecology}

520 Having measured both spatial and social behaviour, statistical approaches must incorporate both 521 data types to compare their effects and/or to ensure they are accounted for when investigating

522 disease dynamics. Controlling for space is a long-standing consideration in ecology (Tobler, 523 1970), so there is no shortage of methods for dealing with spatial structuring. The challenge, 524 then, is incorporating these data into the node-and-edge structure of social network data 525 (Manlove et al., 2018; Mourier et al., 2019; Silk, Croft, Delahay, Hodgson, Boots, et al., 2017), 526 or vice versa (Andris, 2016; Mourier et al., 2019). Modelling approaches should take two main

527 forms: investigating the relationship between space and social network structure, and 528 investigating the extent to which space and/or sociality explains variation in disease (or vice $529 v e r s a)$. These analyses may take several formats: network-level, dyadic, or node-level (Figure 2, 530 Section 3). The list of network methods we provide is by no means exhaustive, but represents an 531 indicative selection of methods that can be used for spatial-social analysis (Silk, Croft, Delahay, 532 Hodgson, Boots, et al., 2017). For each method, we reference packages or tutorials that can help 533 to carry out the analyses; however, these examples are similarly non-comprehensive, and 534 researchers may seek out and use alternative software in many cases.

\section{Considering spatial confounding with network permutations}

536 In network ecology, spatial structuring is commonly controlled for by permuting the observed 537 data in a way that maintains the spatial activity of individuals but randomises their social 538 behaviour. These permutations can either be done at the level of the datastream (e.g. randomly 539 swapping individuals' memberships within social groups, but only allowing swaps within the 540 same locations; Farine et al., 2015) or at the network-level (e.g. randomly re-assigning the social 541 network positions of individuals observed in the same place as one another; Firth \& Sheldon, 
542 2016). Following the creation of the null networks, any given statistic of interest can then be

543 calculated from them, and the distribution of this statistic expected under spatial structure alone

544 can be generated (Whitehead, 2008). If the same statistic in the observed social network is

545 statistically different from this value, it demonstrates a significant effect above any spatial

546 structuring. This methodology has proven useful for differentiating spatial and social processes,

547 notably in great tits, where individuals' social associations during winter foraging determine

548 subsequent spatial decisions during breeding (Firth \& Sheldon, 2016), even more so than

549 expected given winter ranges. Such null network models can be constructed using e.g. the

550 ‘asnipe` package (Farine, 2013). In a similar sense, "spatially embedded" network models can be

551 used to investigate whether spatial effects can explain social structuring (Daraganova et al.,

552 2012), or spatial measures can be used in concert with contact patterns to derive spatially

553 controlled dyadic traits (Davis et al., 2015), e.g. using the residuals of correlations between

554 spatial and social measures (Whitehead \& James, 2015).

Just as 'null social networks' can be created through permuting social behaviour, researchers can create null spatial models (Figure 2) by permuting individuals' spatial activity within the observed dataset while keeping other elements constant. Such methods may aid in comparing the emergent social network to the observed data to investigate whether individuals are actively interacting with (or avoiding) each other, potentially providing insights for disease (Perony, Tessone, König, \& Schweitzer, 2012; Richardson \& Gorochowski, 2015; Spiegel, Leu, Sih, \&

562 Bull, 2016; Woodroffe et al., 2016).

Similarly, permutation can be carried out at any level of the data processing to allow specific null 565 hypothesis testing, whereby particular aspects of the data are controlled for while other aspects are allowed to be randomised. For instance, a permutation may swap the observations within the

567 raw data, or the edges between the nodes in the derived network, or the nodes themselves

568 (Whitehead 2008). In this way, each test comes with its own null hypothesis, and conclusions

569 should be drawn in relation to this hypothesis. For instance, previous studies have noted that

570 permuting the node-level characteristics may be more suited for examining null hypotheses

571 surrounding specific behaviours (Firth et al. 2018) as permuting the raw data under standard

572 datastream permutations only allows for assessing null hypotheses which assume that many 
573 aspects of sociality (such as individual variation in social propensity) are random processes (and

574 thus hold different levels of variation than observed in the real system). <Josh add some

575 sentences about the different levels that permutations can occur at (SS says "node, dyadic and

576 global levels")>.

577

578 Furthermore, despite the well-understood nature of network permutations and their widespread

579 use in network ecology, their utility mainly lies in gauging the evidence for the contributions of

580 spatial or social behaviour, rather than accurately gaining estimates of the contribution of both

581 behaviours to a given (disease) phenotype in the form of an effect size (Franks et al $2020 \mathrm{MEE}$ ).

582 This is crucial, because (as discussed above) there are many situations in which quantifying

583 spatial effects and directly comparing them with sociality effects is an important component of a

584 study design - for example, where a study aims to identify transmission mechanisms, density

585 dependence, or susceptibility effects (see Section 3). For all such analyses, researchers will likely

586 benefit from approaches that can provide interpretable effect estimates of some sort for both

587 spatial and social behaviours. Similarly, there are specific spatial questions that require

588 alternative spatial analyses: for example, researchers may want to quantify the two-dimensional

589 landscape of network structure, which requires specialised analytical constructs other than

590 standard permutations (Albery, Morris, et al., 2020). All approaches we outline below will

591 provide one or more such pieces of information, allowing greater analytical flexibility, and

592 facilitating a wider range of spatial-social questions. However, it is also noted that each of them

593 can be combined with data permutation tests if deemed useful or necessary, where the tests

594 below can be rerun on different permutations of the observed dataset. Such an approach may, for

595 instance, be useful for initial tests of assurance in these different kinds of tests (e.g. for

596 examining whether the reported test statistics differ from those generated using randomised

597 datasets), for comparing the abilities of different methods, or for drawing general predictions

598 about the dynamics of particular diseases (and our estimates of them) under different

599 reconfigurations of the observed social network (e.g. as done for COVID19 in a real-world

600 human social network - Firth et al. 2020). 


\section{Edge-level analyses}

602 Disease analyses commonly aim to investigate how network structure affects pathogen

603 transmission or, reciprocally, how infections alter the network's topology (Craft, 2015; Sah et al.,

604 2018; White et al., 2017). In many cases, multiple spatial and social networks may be necessary

605 to provide clarity on the processes at work: for example, does infection alter the frequency of

606 contact events directly, or does it alter individuals' movements in space, with knock-on effects

607 on the contact network?

608 Dyadic models

609 Social, spatial, and disease data commonly comprise pairwise traits between individuals (e.g.

610 distance matrices or pathogen sharing; see Figure 2, Section 2) many of which resist being coded

611 as node-level traits. Analyses that investigate relationships among these data are problematic

612 because similarity matrices are fraught with non-independence: most notably, each row/column

613 represents a replicated individual. Not correcting for this non-independence will inflate the

614 significance of the effects detected, potentially biasing inference. There are a number of

615 specialised ways to deal with non-independence when correlating dyadic data. For example,

616 Mantel tests and Multiple Regression Quadratic Assignment Procedures (MRQAP) produce

617 conservative correlation coefficient estimates and $p$-values through matrix permutations (e.g.

618 VanderWaal, Atwill, Isbell, \& McCowan, 2014), and can be carried out using the `asnipe`

619 package (Farine, 2013). Generalised Dissimilarity Models (GDMs) are designed specifically to

620 analyse dyadic data while accounting for non-independence and non-linearities in the data, e.g.

621 when quantifying the relative importance of spatial and social proximity in driving viral

622 transmission in lions (Fountain-Jones et al., 2017). The R package `gdm` will implement them

623 (Manion et al., 2018). Finally, multi-membership random effects can be employed to accurately

624 quantify the importance of node-level traits relative to pairwise interactions (Rushmore et al.,

625 2013), and can be carried out using the packages `MCMCglmm` (Hadfield, 2010) and `mgcv'

626 (Wood, 2011). 
628 Representing a more complex variation on the theme of dyadic analyses, Latent Space Models 629 (LSMs) and Exponential Random Graph Models (ERGMs) are versatile tools that model edge-

630 level traits as response variables, incorporating both edge- and node-level traits as explanatory 631 variables (Sewell \& Chen, 2015; Silk, Croft, Delahay, Hodgson, Weber, et al., 2017; see Silk \& 632 Fisher, 2017 for a guide). These variables could include both dyadic spatial/social proximity 633 metrics and individual parasitism, allowing testing of spatial/social components of transmission.

634 Both classes of models can be conceptualised as network-specific adaptations of GLMs, but they 635 differ in the ways they model network structure, and in the process of model fitting (Silk, Croft, 636 Delahay, Hodgson, Weber, et al., 2017; Silk \& Fisher, 2017). Importantly, ERGMs may be 637 poorly suited to association-based networks unless sampling biases are absent or well-accounted 638 for (Silk, Croft, Delahay, Hodgson, Weber, et al., 2017; Silk \& Fisher, 2017). LSMs and ERGMs 639 can be constructed using `latentnet` (Shortreed, Handcock, \& Hoff, 2006) and `ergm` (Hunter, 640 Handcock, Butts, Goodreau, \& Morris, 2008), respectively.

\section{Node-level analyses}

642 Network analyses may use node-level traits derived from the social network as response or 643 explanatory variables in statistical models. Below, we outline some ways to control for spatial 644 autocorrelation in network analyses of disease. These models can investigate spatial structuring 645 of social network-derived traits, or estimate spatial processes alongside links between social 646 behaviour and disease.

\section{Spatial autocorrelation variance components}

648 Hierarchical statistical models (i.e., Generalised Linear Mixed Models, or GLMMs) can control 649 for spatial autocorrelation with variance components (random effects), using individuals' point 650 locations to estimate and control for spatial covariance. The analytical workflow for spatial 651 autocorrelation models involves adding the autocorrelation term and comparing it to the base 652 model to investigate whether it changes model fit, accounts for substantial variance, and/or alters 653 fixed effect estimates. In so doing, the spatial effect will account for spatial variation in social 654 behaviour whether sociality is a response or explanatory variable, presenting a good hold-all for 
655 spatial-social disease analyses. Autocorrelation functions include row/column effects (Stopher et

656 al., 2012), wherein individual $\mathrm{X}$ and Y coordinates (e.g. latitude/longitude) are fitted as

657 discretised integer values connected by autoregressive processes. Such formulations can be

658 computationally intensive, but modern methods such as the stochastic partial differentiation

659 equation (SPDE) in the Integrated Nested Laplace Approximation (INLA) approach are fast,

660 flexible, and increasing in popularity (Lindgren, Rue, \& Lindstrom, 2011; see

661 https://ourcodingclub.github.io/2018/12/04/inla.html for a tutorial). Similar flexible spatial

662 effects can be fitted in Generalised Additive (Mixed) Models (GAMMs), by fitting a tensor

663 smoothing function to individuals' continuous $X$ and Y coordinates. See

664 https://noamross.github.io/gams-in-r-course/ for a tutorial. Available R packages include `mgcv`

665 (Wood, 2011) and 'INLA' (Lindgren \& Rue, 2015).

666 Fitting dyadic associations in node-level analyses

667 Dyadic variance components offer a useful alternative to point-location-based autocorrelation

668 functions, particularly because they allow easy mixing of node-level and dyadic traits in familiar

669 statistical models. Quantitative genetic analyses commonly fit a square matrix of genetic

670 relatedness in the variance component of an "animal model" to estimate genetic heritability in

671 the response variable (Kruuk, 2004). Because these models allow the fitting of multiple such

672 matrices, the models have been supplemented with home range overlap matrices (Stopher et al.,

673 2012). This approach allows extrication of environmental and genetic sources of variation, and

674 can be extended to use social association matrices (Frere et al., 2010; Thomson, Winney, Salles,

$675 \&$ Pujol, 2018) to differentiate spatial and social contributions to a given phenotype. For

676 example, do individuals that associate more often have more similar pathogen intensities? Does

677 this result hold when space sharing is accounted for (Webber \& Vander Wal, 2018)? These

678 models can be carried out in linear modelling packages including 'MCMCglmm` (Hadfield,

679 2010), 'ASReml' (Gilmour, Gogel, Cullis, \& Thompson, 2009), and 'INLA' (Holand,

680 Steinsland, Martino, \& Jensen, 2013).

\section{Considering analytical timescales}

682 The selection of an appropriate timescale is often a necessity of spatial-social analyses, and many

683 available frameworks for spatial-social analysis struggle with incorporating temporal 
684 dependence. The choice of analytical timescale can have dramatic effects on a study's

685 conclusions: for example, Springer, Kappeler, \& Nunn (2017) simulated environmental and

686 direct transmission of gastrointestinal parasites in a lemur population, finding that dynamic

687 networks resulted in larger outbreaks than static equivalents. The options for spatial timescale are

688 numerous: a study could use nest or burrow locations to study distributions of vector-borne

689 parasites (Wood et al., 2007) or to investigate whether distance and infection correlate (Bull et

690 al., 2012), or researchers could link chronic parasite infections with an individual's average

691 location over a predetermined timescale - e.g., the previous year (Albery et al., 2019). Landscape

692 structure and climatic conditions can interact with time-dependent habitat selection behaviours,

693 creating spatiotemporal coincidence of individuals and thereby encouraging social associations.

694 Within each study system, researchers need to establish which time periods should be used to

695 summarise an individual's spatial movements and social interactions, and how these behaviours

696 apply to pathogens of varying infectious periods and development times.

698 Crucially, associations through spatial behaviour can transcend time: that is, individuals can have 699 meaningfully overlapping home ranges even if they were never alive at the same time (Jacoby \&

700 Freeman, 2016). In contrast, social contact requires spatiotemporal coincidence (Manlove et al.,

701 2018; Whitehead, 2008). Spatial behaviours' time-independence could be a positive or a

702 negative, depending on the question to hand, and researchers must consider the timescale of the

703 pathogen. For example, space use combined with a temporal delay may be the best way to

704 describe transmission of certain parasites, but not others (Gilbertson et al., 2020; Manlove et al.,

705 2018; Richardson \& Gorochowski, 2015). Furthermore, if local environmental variation is stable

706 over long time periods and influences disease risk, spatial associations may predict disease

707 similarity even in the absence of any possible social contacts (i.e. across non-temporally-

708 overlapping generations). This knowledge could inform which behaviours could be important

709 when modelling transmission dynamics - and, conversely, comparing the importance of

710 (temporally lagged) spatial and social behaviours could illuminate the transmission modes or

711 epidemiological dynamics of a given pathogen (e.g. Albery, Newman, et al., 2020; Springer et

712 al., 2017; see Section 3C). 
714 The repeatability of behaviour (sometimes conceptualised as "personality") is an important,

715 rapidly developing area of research (Dingemanse \& Dochtermann, 2013; Moirón, Laskowski, \&

716 Niemelä, 2019) which is also often considered for movement behaviours (Jacoby \& Freeman,

717 2016; Webber et al., 2020; Webber \& Vander Wal, 2018) or social behaviours (Aplin et al. 2015;

718 Firth et al. 2017; Krause et al. 2017). If behaviour is highly repeatable across time, e.g. where

719 individuals inhabit similar home ranges from year to year (Stopher et al., 2012), timescale

720 problems may be somewhat avoidable. This will also depend on the pathogen of interest:

721 environmental parasites may have more constant spatial hotspots driven by consistent climatic

722 factors, so that lifetime home ranges capture substantial variation in parasitism; meanwhile,

723 directly transmitted parasites may exhibit waves of transmission across the population, such that

724 spatial hotspots are more ephemeral and a restricted analytical timescale is vital. Fortunately,

725 many of the analytical frameworks we describe are able to incorporate temporal structures: for

726 example, INLA can fit fluctuating spatiotemporal fields across years and seasons (Albery et al.,

727 2019), and temporal ERGMs (tERGMs) can handle changing network structures through time

728 (Silk, Croft, Delahay, Hodgson, Weber, et al., 2017). Thus, even the enduring problem of

729 timescale selection is solvable when interactions between environment, movement, sociality, and

730 parasitism are understood and analysed properly.

\section{6. Synthesis and future directions}

732 We have so far provided a guide to carrying out spatial-social network analysis in disease

733 ecology, from conception through to analysis. In this section, we discuss ideal empirical systems

734 for addressing spatial-social questions, and we detail potential benefits emerging from the

735 unification of spatial and social analysis.

\section{Model systems}

737 Meta-analysis is a promising option for large-scale investigation of spatial-social influences in

738 disease ecology. The number of published social network analyses has increased exponentially in

739 recent years (Webber \& Vander Wal, 2019), and repositories of network data are becoming

740 available as a result (Sah, Méndez, \& Bansal, 2019). These resources can help to compensate for

741 the lack of cross-system synthesis in this field so far. By analysing contact data alongside spatial 
742 behaviour across the published literature, we can ask broadly informative questions such as: how

743 many social network analyses include spatial data? How often are space and sociality highly

744 correlated? How might this impact studies' findings? Such analyses may identify general

745 indicators of when and where to be concerned about space for social network analyses (and even

746 for disease ecology studies in general), as well as potentially testing the criteria laid out in this

747 review. Furthermore, even if pathogen data are not available for the large majority of spatial-

748 social network datasets, empirically parameterised simulations of disease spread within a meta-

749 analytical framework (e.g. Sah et al., 2018) could be a useful tool for gaining a general

750 understanding of how spatial and social drivers of disease can be untangled, and which kinds of

751 systems and network structures best allow this separation.

753 Many empirical systems lend themselves to spatial-social analysis. Fundamentally, any system

754 with extricable/tractable social and spatial behaviour could be used for such analyses, and

755 fission-fusion social systems may be especially well-suited for this reason: censuses and GPS

756 records can regularly identify individuals' group memberships separately alongside their spatial

757 locations, allowing untangling of spatial-social associations (Box 1). Such systems include many

758 well-studied animals, such as dolphins (Lusseau et al., 2006), great tits (Firth \& Sheldon, 2016),

759 and deer (Stopher et al., 2012). Ants likewise represent a promising model system for this

760 reason: using motion-tracking cameras, spatial behaviour can be tracked and then social contacts

761 extricated (Modlmeier et al., 2019; Stroeymeyt et al., 2018): for example, trophallaxis or

762 physical touch events can be used to create a contact network, while space use distributions or

763 movement trajectories are used to characterise their spatial behaviour. Although the two will

764 correlate, there is likely to be considerable testable variation: that is, of the ants that overlap in

765 space with one another, only a subset of dyads will give or receive trophallaxis to each other

766 (Modlmeier et al., 2019). Ants' social networks respond predictably to spatial changes

767 (Modlmeier et al., 2019) and pathogen presence (Stroeymeyt et al., 2018), with group-level

768 trends emerging from predictable individual-level behaviours, lending them well to high-

769 resolution movement models.

770

771 Knowledge of a wide range of different pathogens is a further advantage for a potential study

772 system, particularly because this may allow testing of the spatial-social continuum that we 
773 outlined in the pathogen transmission section above. Rodents are some of the best-studied model

774 systems for disease ecology, yet because rodents are generally too small for battery-powered

775 high-resolution GPS tracking, the tools available for studying their spatial behaviour at high

776 resolution in the wild are limited. To fill this gap, the development of lightweight bluetooth

777 technology has facilitated the use of highly sensitive proximity loggers in wild Mastomys mice

778 (Berkvens, Olivares, Mercelis, Kirkpatrick, \& Weyn, 2019). Using environmentally placed

779 loggers with wide ranges and extended battery lives, it is possible to collect regular spatial

780 locations alongside social contact data, providing an exciting model system with which to

781 investigate space and sociality simultaneously (Berkvens et al., 2019). This methodology could

782 be combined with the considerable literature on trapping-based contact networks in field voles

783 (Davis et al., 2015; Wanelik, 2019) and other rodents (e.g. Grear et al., 2009). Notably, sleepy

784 lizards (Tiliqua rugosa) have recently been proposed as an ideal system for the integration of

785 social and spatial analyses, particularly focussing on ectoparasite transmission, and with many

786 exciting future opportunities for joint spatial-social analyses (Sih et al., 2018). As such, the list of

787 potential systems is phylogenetically diverse and extremely promising, with many opportunities

788 for further specialisation under this umbrella.

789 Connecting environmental, animal, and human health with spatial-social

790 analyses

791 Unlike human systems where linking real-world disease dynamics to real-world social contact

792 networks is exceptionally rare despite much interest (Firth et al. 2020), there is a great number of

793 real-world social contact network monitoring efforts from natural animal systems (Sah et al.

794 2017; Sah et al. 2019) meaning these hold unique potential to contribute to understanding

795 broader societal issues relating to disease spread and health. Specifically, aside from

796 strengthening inference and improving model accuracy, the potential practical benefits of unified

797 spatial-social analysis for disease ecology are numerous. Integration will improve our ability to

798 investigate transmission mechanisms and density dependence, while conveying operational

799 benefits (Section 3). Furthermore, better empirical understanding will inform the relevant

800 spatiotemporal scales of transmission dynamics, providing parameters for scalable models of

801 spatial movement that implicitly or explicitly account for social contact-driven transmission 
802 events within them (White, Forester, \& Craft, 2018). Building on rapidly developing interest in

803 disease-behaviour-network feedbacks ( Section 3A), spatial-social analyses could integrate

804 existing models of spatial-social feedback (e.g. Firth \& Sheldon, 2016) with those that identify

805 reciprocal changes in network topology in response to disease transmission (e.g. Stroeymeyt et

806 al., 2018).

807

808 All such endeavours will help to predict how altered behaviour will affect disease transmission

809 (and vice versa) in the wake of large-scale community perturbations. This includes short-term

810 events (e.g. zoonotic outbreaks or catastrophic events), long-term trends (e.g. climate change-

811 induced alterations to global transport systems), or behavioural animal health interventions (e.g.

812 translocations), all of which will alter contact patterns separately from spatial movements. For

813 example, individual variability in raccoon ranging behaviour can reduce the effectiveness of

814 rabies vaccination interventions (McClure, Gilbert, Chipman, Rees, \& Pepin, 2020).

815 Understanding how landscape structure alters raccoons' spatial behaviour, and therefore disease

816 spread, will help to anticipate geographic variation in intervention success. As another example,

817 it is well established that culling British badgers (Meles meles) is an ineffective method of

818 control for bovine tuberculosis (Mycobacterium bovis). The culling-associated disruption of local

819 population structure provokes badgers to disperse, moving further than they otherwise would and

820 making more social contacts in the process (Carter et al., 2007; Ham, Donnelly, Astley, Jackson,

821 \& Woodroffe, 2019; Tuyttens et al., 2000). As such, this perturbation of the social network

822 induces a spatial movement, which is expected to result in a subsequent rearrangement of the

823 social contact network. These changes in network structure may facilitate $M$. bovis spread across

824 the countryside, directly contravening the intended control efforts by infecting cattle in

825 surrounding areas (Donnelly et al., 2007). This example is hard to conceptualise without

826 considering the social and spatial networks in tandem, as well as considering the landscape itself.

827 Under rapid ongoing global change, a proper understanding of the links between the

828 environment, animal movement, and social behaviour will be crucial for understanding how

829 disruptions and natural disasters such as fires, floods, and hurricanes will impact wildlife disease

830 (Silk et al., 2019). Studies have already connected ongoing ecological tragedies such as fire with

831 animal movement and one health consequences (Bonilla-Aldana et al., 2019), and spatial-social

832 analysis is set to be an invaluable tool for anticipating and combatting their effects. 


\section{Acknowledgements}

834 This work was supported by NSF Grant No. 1414296 as part of the joint NSF-NIH-USDA

835 Ecology and Evolution of Infectious Diseases program. JAF was supported by a research

836 fellowship from Merton College and BBSRC (BB/S009752/1) and acknowledges funding from

837 NERC (NE/S010335/1). We would like to thank Matt Silk, Sebastian Sosa, two anonymous

838 reviewers, and the associate editor for their helpful comments.

839

840

Adams, J., Faust, K., \& Lovasi, G. S. (2012). Capturing context: Integrating spatial and social network analyses. Social Networks, 34(1), 1-5. doi: 10.1016/j.socnet.2011.10.007

Albery, G. F., Becker, D. J., Kenyon, F., Nussey, D. H., \& Pemberton, J. M. (2019). The finescale landscape of immunity and parasitism in a wild ungulate population. Integrative and Comparative Biology, icz016(5), 1-11. doi: 10.1093/icb/icz016

Albery, G. F., Morris, A., Morris, S., Pemberton, J. M., Clutton-, T. H., Nussey, D. H., \& Firth, J. A. (2020). Spatial point locations explain a range of social network positions in a wild ungulate. BioRxiv. doi: https://doi.org/10.1101/2020.06.04.135467

Albery, G. F., Newman, C., Ross, J. G. B., Macdonald, D. W., Bansal, S., \& Buesching, C. D. (2020). Negative density-dependent parasitism in a group-living carnivore.

Andris, C. (2016). Integrating social network data into GISystems. International Journal of Geographical Information Science, 30(10), 2009-2031. doi:

10.1080/13658816.2016.1153103

Antonovics, J. (2017). Transmission dynamics: Critical questions and challenges. Philosophical Transactions of the Royal Society B: Biological Sciences, 372(1719). doi:

10.1098/rstb.2016.0087

Armansin, N. C., Stow, A. J., Cantor, M., Leu, S. T., Klarevas-Irby, J. A., Chariton, A. A., \& Farine, D. R. (2019). Social Barriers in Ecological Landscapes: The Social Resistance Hypothesis. Trends in Ecology and Evolution, $x x(\mathrm{xx}), 1-12$. doi: 10.1016/j.tree.2019.10.001 Becker, D. J., Albery, G. F., Kessler, M. K., Lunn, T. J., Falvo, C. A., Czirják, G. Á., ... Plowright, R. K. (2020). Macroimmunology: the drivers and consequences of spatial 
patterns in wildlife immune defense. Journal of Animal Ecology, 89(4), 972-995. doi: $10.1111 / 1365-2656.13166$

Becker, D. J., Czirják, G. Á., Volokhov, D. V., Bentz, A. B., Carrera, J. E., Camus, M. S., ... Streicker, D. G. (2018). Livestock abundance predicts vampire bat demography, immune

Becker, D. J., Nachtmann, C., Argibay, H. D., Botto, G., Escalera-Zamudio, M., Carrera, J. E., ... Streicker, D. G. (2019). Leukocyte Profiles Reflect Geographic Range Limits in a Widespread Neotropical Bat. Integrative and Comparative Biology, icz007. doi: 10.1093/icb/icz007

Becker, D. J., Streicker, D. G., \& Altizer, S. (2015). Linking anthropogenic resources to wildlifepathogen dynamics: a review and meta-analysis. Ecology Letters, 18(5), n/a-n/a. doi: 10.1111/ele.12428

Berkvens, R., Olivares, I. H., Mercelis, S., Kirkpatrick, L., \& Weyn, M. (2019). Contact Detection for Social Networking of Small Animals. In Advances on P2P, Parallel, Grid, Cloud and Internet Computing (pp. 405-414). doi: 10.1007/978-3-030-02607-3_37

Bonilla-Aldana, D. K., Suárez, J. A., Franco-Paredes, C., Vilcarromero, S., Mattar, S., GómezMarín, J. E., ... Rodríguez-Morales, A. J. (2019). Brazil burning! What is the potential impact of the Amazon wildfires on vector-borne and zoonotic emerging diseases? - A statement from an international experts meeting. Travel Medicine and Infectious Disease, 31, 12-15. doi: 10.1016/j.tmaid.2019.101474

Bradley, J. E., \& Jackson, J. A. (2008). Measuring immune system variation to help understand host-pathogen community dynamics. Parasitology, 135(7), 807-823. doi:

\subsection{7/S0031182008000322}

Broekhuis, F., Madsen, E. K., Keiwua, K., \& Macdonald, D. W. (2019). Using GPS collars to investigate the frequency and behavioural outcomes of intraspecific interactions among carnivores: A case study of male cheetahs in the Maasai Mara, Kenya. PLOS ONE, 14(4), e0213910. doi: 10.1371/journal.pone.0213910

Bull, C. M., Godfrey, S. S., \& Gordon, D. M. (2012). Social networks and the spread of Salmonella in a sleepy lizard population. Molecular Ecology, 21(17), 4386-4392. doi: 10.1111/j.1365-294X.2012.05653.x 
Carter, S. P., Delahay, R. J., Smith, G. C., Macdonald, D. W., Riordan, P., Etherington, T. R., ... Cheeseman, C. L. (2007). Culling-induced social perturbation in Eurasian badgers Meles meles and the management of TB in cattle: An analysis of a critical problem in applied ecology. Proceedings of the Royal Society B: Biological Sciences, 274(1626), 2769-2777. doi: $10.1098 / \mathrm{rspb} .2007 .0998$

Castles, M., Heinsohn, R., Marshall, H. H., Lee, A. E. G., Cowlishaw, G., \& Carter, A. J. (2014). Social networks created with different techniques are not comparable. Animal Behaviour, 96, 59-67. doi: 10.1016/j.anbehav.2014.07.023

Clutton-Brock, T. H. (1974). Primate social organisation and ecology. Nature, 250(5467), 539_ 542. doi: $10.1038 / 250539 \mathrm{a} 0$

Craft, M. E. (2015). Infectious disease transmission and contact networks in wildlife and livestock. Philosophical Transactions of the Royal Society B: Biological Sciences, 370(1669), 20140107-20140107. doi: 10.1098/rstb.2014.0107

Croft, D. P., James, R., \& Krause, J. (2008). Exploring animal social networks. Retrieved from https://press.princeton.edu/books/paperback/9780691127521/exploring-animal-socialnetworks

Crook, J. H. (1964). The Evolution of Social Organisation and Visual Communication in the Weaver Birds (Ploceinae).

Crook, J. H. (1970). Social organization and the environment: Aspects of contemporary social ethology. Animal Behaviour, 18, 197-209. doi: 10.1016/S0003-3472(70)80029-X

Crook, J. H., \& Gartlan, J. S. (1966). Evolution of Primate Societies. Nature, 210(5042), 12001203. doi: $10.1038 / 2101200 \mathrm{a} 0$

Daraganova, G., Pattison, P., Koskinen, J., Mitchell, B., Bill, A., Watts, M., \& Baum, S. (2012). Networks and geography: Modelling community network structures as the outcome of both spatial and network processes. Social Networks, 34(1), 6-17. doi: 10.1016/J.SOCNET.2010.12.001

Davis, S., Abbasi, B., Shah, S., Telfer, S., Begon, M., \& Davis, S. (2015). Spatial analyses of wildlife contact networks. Journal of the Royal Society, Interface / the Royal Society, 12(102), 20141004. doi: 10.1098/rsif.2014.1004

Dingemanse, N. J., \& Dochtermann, N. A. (2013). Quantifying individual variation in behaviour: Mixed-effect modelling approaches. Journal of Animal Ecology, 82(1), 39-54. doi: 
924 Donnelly, C. A., Wei, G., Johnston, W. T., Cox, D. R., Woodroffe, R., Bourne, F. J., ...

925 Morrison, W. I. (2007). Impacts of widespread badger culling on cattle tuberculosis:

926 concluding analyses from a large-scale field trial. International Journal of Infectious

927 Diseases : IJID : Official Publication of the International Society for Infectious Diseases,

928 11(4), 300-308. doi: 10.1016/j.ijid.2007.04.001

929 Dougherty, E. R., Seidel, D. P., Carlson, C. J., Spiegel, O., \& Getz, W. M. (2018). Going through

930 the motions : incorporating movement analyses into disease research. Ecology Letters,

$931 \quad 21(4), 588-604$. doi: 10.1111/ele.12917

932 Ezenwa, V. O., Archie, E. A., Craft, M. E., Hawley, D. M., Martin, L. B., Moore, J., \& White, L.

933 (2016). Host behaviour-parasite feedback: an essential link between animal behaviour and

934 disease ecology: Table 1. Proceedings of the Royal Society B: Biological Sciences,

935 283(1828), 20153078. doi: 10.1098/rspb.2015.3078

936 Ezenwa, V. O., Ghai, R. R., McKay, A. F., \& Williams, A. E. (2016). Group living and pathogen infection revisited. Current Opinion in Behavioral Sciences, 12, 66-72. doi: 10.1016/j.cobeha.2016.09.006

Farine, D. R. (2013). Animal social network inference and permutations for ecologists in R using asnipe. Methods in Ecology and Evolution, 4(12), 1187-1194. doi: 10.1111/2041-

942 Farine, D. R. (2015). Proximity as a proxy for interactions: issues of scale in social network analysis. Animal Behaviour, 104, e1-e5. doi: 10.1016/j.anbehav.2014.11.019

Farine, D. R., Firth, J. A., Aplin, L. M., Crates, R. A., Culina, A., Garroway, C. J., ... Sheldon, B. C. (2015). The role of social and ecological processes in structuring animal populations: a case study from automated tracking of wild birds. Royal Society Open Science, 2(4), 150057-150057. doi: 10.1098/rsos.150057

Firth, J. A., \& Sheldon, B. C. (2015). Experimental manipulation of avian social structure reveals segregation is carried over across contexts. Proceedings of the Royal Society B: Biological Sciences, 282(1802). doi: 10.1098/rspb.2014.2350

953 Firth, J. A., Voelkl, B., Crates, R. A., Aplin, L. M., Biro, D., Croft, D. P., \& Sheldon, B. C. 
(2017). Wild birds respond to flockmate loss by increasing their social network associations to others. Proceedings of the Royal Society B: Biological Sciences, 284(1854). doi:

Fountain-Jones, N. M., Packer, C., Troyer, J. L., VanderWaal, K., Robinson, S., Jacquot, M., \& Craft, M. E. (2017). Linking social and spatial networks to viral community phylogenetics reveals subtype-specific transmission dynamics in African lions. Journal of Animal Ecology, 86(6), 1469-1482. doi: 10.1111/1365-2656.12751

Frere, C. H., Krutzen, M., Mann, J., Connor, R. C., Bejder, L., \& Sherwin, W. B. (2010). Social and genetic interactions drive fitness variation in a free-living dolphin population. Proceedings of the National Academy of Sciences, 107(46), 19949-19954. doi: 10.1073/pnas.1007997107

Gilbertson, M. L. J., White, L. A., \& Craft, M. E. (2020). Trade-offs with telemetry-derived contact networks for infectious disease studies in wildlife. Methods in Ecology and Evolution, 2041-210X.13355. doi: 10.1111/2041-210X.13355

Gilmour, A. R., Gogel, B. J., Cullis, B. R., \& Thompson, R. (2009). ASReml User Guide. Retrieved from https://www.vsni.co.uk/downloads/asreml/release3/UserGuide.pdf

Grear, D. A., Perkins, S. E., \& Hudson, P. J. (2009). Does elevated testosterone result in increased exposure and transmission of parasites? Ecology Letters, 12(6), 528-537. doi:

Ham, C., Donnelly, C. A., Astley, K. L., Jackson, S. Y. B., \& Woodroffe, R. (2019). Effect of 10.1111/j.1461-0248.2009.01306.x

Hadfield, J. D. (2010). MCMC methods for multi-response generalized linear mixed models: the MCMCglmm R package. Journal of Statistical Software, 33(2), 1-22. doi: culling on individual badger Meles meles behaviour: Potential implications for bovine tuberculosis transmission. Journal of Applied Ecology, 56(11), 2390-2399. doi: $10.1111 / 1365-2664.13512$

Hamede, R. K., Bashford, J., McCallum, H., \& Jones, M. (2009). Contact networks in a wild Tasmanian devil (Sarcophilus harrisii) population: Using social network analysis to reveal seasonal variability in social behaviour and its implications for transmission of devil facial tumour disease. Ecology Letters, 12, 1147-1157. doi: 10.1111/j.1461-0248.2009.01370.x Hawley, D. M., Etienne, R. S., Ezenwa, V. O., \& Jolles, A. E. (2011). Does animal behavior 
underlie covariation between hosts' exposure to infectious agents and susceptibility to infection? Implications for disease dynamics. Integrative and Comparative Biology, 51(4), 528-539. doi: 10.1093/icb/icr062

He, P., Maldonado-chaparro, A. A., \& Farine, D. R. (2019). The role of habitat configuration in shaping social structure: a gap in studies of animal social complexity. Behavioral Ecology and Sociobiology, 73(9).

Holand, A. M., Steinsland, I., Martino, S., \& Jensen, H. (2013). Animal Models and Integrated

Jacoby, D. M. P., Papastamatiou, Y. P., \& Freeman, R. (2016). Inferring animal social networks Nested Laplace Approximations. Genes|Genomes|Genetics, 3(8), 1241-1251. doi: $10.1534 / \mathrm{g} 3.113 .006700$

Hopkins, S. R., Fleming-Davies, A. E., Belden, L. K., \& Wojdak, J. M. (2020). Systematic review of modelling assumptions and empirical evidence: Does parasite transmission increase nonlinearly with host density? Methods in Ecology and Evolution, 11(4), 476-486. doi: 10.1111/2041-210X.13361

Hunter, D. R., Handcock, M. S., Butts, C. T., Goodreau, S. M., \& Morris, M. (2008). ergm: A Package to Fit, Simulate and Diagnose Exponential-Family Models for Networks. Journal of Statistical Software, 24(3), nihpa54860. doi: 10.18637/jss.v024.i03

Hutchings, M. R., Judge, J., Gordon, I. J., Athanasiadou, S., \& Kyriazakis, I. (2006). Use of trade-off theory to advance understanding of herbivore-parasite interactions. Mammal Review, 36(1), 1-16. doi: 10.1111/j.1365-2907.2006.00080.x

Jacoby, D. M. P., \& Freeman, R. (2016). Emerging Network-Based Tools in Movement Ecology. Trends in Ecology and Evolution, 31(4), 301-314. doi: 10.1016/j.tree.2016.01.011 and leadership: Applications for passive monitoring arrays. Journal of the Royal Society Interface, 13(124). doi: 10.1098/rsif.2016.0676

Kays, R., Crofoot, M. C., Jetz, W., \& Wikelski, M. (2015). Terrestrial animal tracking as an eye on life and planet. Science, 348(6240), aaa2478. doi: 10.1126/science.aaa2478

Krause, J., James, R., Franks, D. W., \& Croft, D. P. (2015). Animal social networks. Oxford, UK: Oxford University Press.

Kruuk, L. E. B. (2004). Estimating genetic parameters in natural populations using the "animal model." Philosophical Transactions of the Royal Society B: Biological Sciences, 359(1446), 873-890. doi: 10.1098/rstb.2003.1437 
1016 Leu, S. T., Sah, P., Krzyszczyk, E., Jacoby, A., \& Mann, J. (2020). Sex, synchrony, and skin 1017 contact: integrating multiple behaviors to assess pathogen transmission risk. Behavioral 1018 Ecology, 1-10. doi: 10.1093/beheco/araa002

1019 Lindgren, F., \& Rue, H. (2015). Bayesian Spatial Modelling with R-INLA. Journal of Statistical $1020 \quad$ Software, 63(19), 1-25. doi: 10.18637/jss.v063.i19

1021 Lindgren, F., Rue, H., \& Lindstrom, J. (2011). An explicit link between Gaussian fields and 1022 Gaussian Markov random fields: the stochastic partial differential equation approach. 1023 Journal of the Royal Statistical Society B, 73(4), 423-498.

1024 Lopes, P. C. (2014). When is it socially acceptable to feel sick? Proceedings of the Royal Society 1025 B: Biological Sciences, 281(1788), 20140218-20140218. doi: 10.1098/rspb.2014.0218 1026 Lopes, P. C., Block, P., \& König, B. (2016). Infection-induced behavioural changes reduce 1027 1028 1029 1030 connectivity and the potential for disease spread in wild mice contact networks. Scientific Reports, 6(1), 31790. doi: 10.1038/srep31790

Lopes, P. C., Block, P., Pontiggia, A., Lindholm, A. K., \& König, B. (2018). No evidence for kin protection in the expression of sickness behaviors in house mice. Scientific Reports, $8(1)$, 16682. doi: 10.1038/s41598-018-35174-0

Lusseau, D., Wilson, B., Hammond, P. S., Grellier, K., Durban, J. W., Parsons, K. M., ... Thompson, P. M. (2006). Quantifying the influence of sociality on population structure in bottlenose dolphins. Journal of Animal Ecology, 75(1), 14-24. doi: 10.1111/j.13652656.2005.01013.x

MacIntosh, A. J. J., Jacobs, A., Garcia, C., Shimizu, K., Mouri, K., Huffman, M. A., \& Hernandez, A. D. (2012). Monkeys in the Middle: Parasite Transmission through the Social Network of a Wild Primate. PLoS ONE, 7(12), 15-21. doi: 10.1371/journal.pone.0051144

Manion, G., Lisk, M., Ferrier, S., Nieto-Lugilde, D., Mokany, K., \& Fitzpatrick, M. C. (2018). gdm: Generalized Dissimilarity Modeling. R package version 1.3.11. Retrieved from https://cran.r-project.org/package=gdm ecology of movement and behaviour: a saturated tripartite network for describing animal contacts. Proceedings. Biological Sciences, 285(1887), 20180670. doi: 10.1098/rspb.2018.0670

McClure, K. M., Gilbert, A. T., Chipman, R. B., Rees, E. E., \& Pepin, K. M. (2020). Variation in 
host home range size decreases rabies vaccination effectiveness by increasing the spatial spread of rabies virus. In The Journal of Animal Ecology (Vol. 25). doi: 10.2307/2256344

Modlmeier, A. P., Colman, E., Hanks, E. M., Bringenberg, R., Bansal, S., \& Hughes, D. P. (2019). Ant colonies maintain social homeostasis in the face of decreased density. 1-17.

Moirón, M., Laskowski, K. L., \& Niemelä, P. T. (2019). Individual differences in behaviour explain variation in survival: a meta-analysis. EcoEvoRxiv Preprints. doi:

\subsection{2/OSF.IO/TZ2V8}

Mourier, J., Lédée, E. J. I., \& Jacoby, D. M. P. (2019). A multilayer perspective for inferring spatial and social functioning in animal movement networks. BioRxiv. doi: 10.1101/749085

Nathan, R., Getz, W. M., Revilla, E., Holyoak, M., Kadmon, R., Saltz, D., \& Smouse, P. E. (2008). A movement ecology paradigm for unifying organismal movement research. Proceedings of the National Academy of Sciences, 105(49), 19052-19059.

O’Brien, P. P., Webber, Q. M. R., \& Vander Wal, E. (2018). Consistent individual differences and population plasticity in network-derived sociality: An experimental manipulation of density in a gregarious ungulate. PLoS ONE, 13(3), 1-21. doi: 10.1371/journal.pone.0193425

Ossi, F., Focardi, S., Picco, G. Pietro, Murphy, A., Molteni, D., Tolhurst, B., .. Cagnacci, F. (2016). Understanding and geo-referencing animal contacts: Proximity sensor networks integrated with GPS-based telemetry. Animal Biotelemetry, 4(1), 1-14. doi: $10.1186 / \mathrm{s} 40317-016-0111-\mathrm{x}$

Pawley, M. D. M., \& McArdle, B. H. (2018). Spatial autocorrelation: Bane or Bonus? BioRxiv, 385526. doi: $10.1101 / 385526$

Peignier, M., Webber, Q. M. R., Koen, E. L., Laforge, M. P., Robitaille, A. L., \& Vander Wal, E. (2019). Space use and social association in a gregarious ungulate: Testing the conspecific attraction and resource dispersion hypotheses. Ecology and Evolution, 9(9), 5133-5145. doi: $10.1002 /$ ece 3.5071

Perony, N., Tessone, C. J., König, B., \& Schweitzer, F. (2012). How Random Is Social Behaviour? Disentangling Social Complexity through the Study of a Wild House Mouse Population. PLoS Computational Biology, 8(11). doi: 10.1371/journal.pcbi.1002786

Psorakis, I., Voelkl, B., Garroway, C. J., Radersma, R., Aplin, L. M., Crates, R. A., ... Sheldon, B. C. (2015). Inferring social structure from temporal data. Behavioral Ecology and 
Pullan, R. L., Sturrock, H. J. W., Soares Magalhaes, R. J., Clements, A. C. A., \& Brooker, S. J. (2012). Spatial parasite ecology and epidemiology: a review of methods and applications. Parasitology, 139(14), 1870-1887. doi: 10.1017/S0031182012000698

Richardson, T. O., \& Gorochowski, T. E. (2015). Beyond contact-based transmission networks:

Robitaille, A. L., Webber, Q. M. R., \& Vander Wal, E. (2019). Conducting social network

Rushmore, J., Caillaud, D., Matamba, L., Stumpf, R. M., Borgatti, S. P., \& Altizer, S. (2013). analysis with animal telemetry data: Applications and methods using spatsoc. Methods in Ecology and Evolution, 10(8), 1203-1211. doi: 10.1111/2041-210X.13215 The role of spatial coincidence. Journal of the Royal Society Interface, 12(111). doi: 10.1098/rsif.2015.0705 Social network analysis of wild chimpanzees provides insights for predicting infectious disease risk. Journal of Animal Ecology, 82(5), 976-986. doi: 10.1111/1365-2656.12088

Sah, P., Mann, J., \& Bansal, S. (2018). Disease implications of animal social network structure: A synthesis across social systems. Journal of Animal Ecology, 87(3), 546-558. doi: 10.1111/1365-2656.12786

Sah, P., Méndez, J. D., \& Bansal, S. (2019). A multi-species repository of social networks. Scientific Data, 6(1), 44. doi: 10.1038/s41597-019-0056-Z

Saito, M. U., \& Sonoda, Y. (2017). Symptomatic Raccoon Dogs and Sarcoptic Mange Along an Urban Gradient. EcoHealth, 14(2), 318-328. doi: 10.1007/s10393-017-1233-1

Schlägel, U. E., Signer, J., Herde, A., Eden, S., Jeltsch, F., Eccard, J. A., \& Dammhahn, M. (2019). Estimating interactions between individuals from concurrent animal movements. Methods in Ecology and Evolution, 2019(May), 2041-210X.13235. doi: 10.1111/2041210X.13235

Seidel, D. P., Dougherty, E., Carlson, C., \& Getz, W. M. (2018). Ecological metrics and methods for GPS movement data. International Journal of Geographical Information Science, 32(11), 2272-2293. doi: 10.1080/13658816.2018.1498097

Sewell, D. K., \& Chen, Y. (2015). Latent Space Models for Dynamic Networks. Journal of the American Statistical Association, 110(512), 1646-1657. doi: 10.1080/01621459.2014.988214 
Model for Networks. Methodology: European Journal of Research Methods for the

Behavioral and Social Sciences, 2(1), 24-33. doi: 10.1027/1614-2241.2.1.24

Sih, A., Spiegel, O., Godfrey, S., Leu, S., \& Bull, C. M. (2018). Integrating social networks, animal personalities, movement ecology and parasites: a framework with examples from a lizard. Animal Behaviour, 136, 195-205. doi: 10.1016/j.anbehav.2017.09.008

Silk, M. J., Croft, D. P., Delahay, R. J., Hodgson, D. J., Boots, M., Weber, N., \& McDonald, R. A. (2017). Using Social Network Measures in Wildlife Disease Ecology, Epidemiology, and Management. BioScience, 67(3), 245-257. doi: 10.1093/biosci/biw175

Silk, M. J., Croft, D. P., Delahay, R. J., Hodgson, D. J., Weber, N., Boots, M., \& McDonald, R.

Silk, M. J., \& Fisher, D. N. (2017). Understanding animal social structure: exponential random graph models in animal behaviour research. Animal Behaviour, 132, 137-146. doi: 10.1016/j.anbehav.2017.08.005

Silk, M. J., Hodgson, D., Rozins, C., Croft, D., Delahay, R., Boots, M., \& McDonald, R. (2019). Integrating social behaviour, demography and disease dynamics in network models: applications to disease management in declining wildlife populations. Philosophical Transactions of the Royal Society B, 374, 20180211.

Smouse, P. E., Focardi, S., Moorcroft, P. R., Kie, J. G., Forester, J. D., \& Morales, J. M. (2010). Stochastic modelling of animal movement. Philosophical Transactions of the Royal Society B: Biological Sciences, 365(1550), 2201-2211. doi: 10.1098/rstb.2010.0078

Sosa, S., Sueur, C., \& Puga-Gonzalez, I. (2020). Network measures in animal social network analysis: their strengths, limits, interpretations and uses. Methods in Ecology and Evolution. doi: 10.1111/2041-210x.13366

Spiegel, O., Leu, S. T., Sih, A., \& Bull, C. M. (2016). Socially interacting or indifferent neighbours? Randomization of movement paths to tease apart social preference and spatial constraints. 971-979. doi: 10.1111/2041-210X.12553

Springer, A., Kappeler, P. M., \& Nunn, C. L. (2017). Dynamic vs. static social networks in 

of Animal Ecology, 86(3), 419-433. doi: 10.1111/1365-2656.12617

1142 Stopher, K. V, Walling, C. a, Morris, A., Guinness, F. E., Clutton-brock, T. H., Pemberton, J.

1143 M., \& Nussey, D. H. (2012). Shared spatial effects on quantitative genetic parameters: accounting for spatial autocorrelation and home range overlap reduces estimates of heritability in wild red deer. Evolution; International Journal of Organic Evolution, 66(8), 2411-2426. doi: 10.5061/dryad.jf04r362

Stratford, K., Stratford, S., \& Périquet, S. (2019). Dyadic associations reveal clan size and social network structure in the fission-fusion society of spotted hyaenas. African Journal of Ecology, (August 2018), aje.12641. doi: 10.1111/aje.12641

Stroeymeyt, N., Grasse, A. V, Crespi, A., Mersch, D. P., Cremer, S., \& Keller, L. (2018). Social network plasticity decreases disease transmission in a eusocial insect. 945(November), 941-945. doi: 10.1126/science.aat4793

Thomson, C. E., Winney, I. S., Salles, O. C., \& Pujol, B. (2018). A guide to using a MultipleMatrix animal model to disentangle genetic and nongenetic causes of phenotypic variance. PLoS ONE, 13(10), e0197720. doi: 10.1371/journal.pone.0197720

Tobler, W. R. (1970). A Computer Movie Simulating Urban Growth in the Detroit Region. Economic Geography, 46, 234. doi: 10.2307/143141

Tuyttens, F. a M., Delahay, R. J., MacDonald, D. W., Cheeseman, C. L., Long, B., \& Donnelly, C. a. (2000). Spatial perturabtion caused by a badger (Meles meles) culling operation: implication for the function of territoriality and the control of bovine tuberculosis (Mycobacterium bovis. Journal of Applied Ecology, 69(0), 815-828.

VanderWaal, K. L., Atwill, E. R., Isbell, L. A., \& McCowan, B. (2014). Linking social and pathogen transmission networks using microbial genetics in giraffe (Giraffa camelopardalis). Journal of Animal Ecology, 83(2), 406-414. doi: 10.1111/13652656.12137

Wanelik, K. (2019). How to characterise shared space use networks.

Webber, Q. M. R., Laforge, M. P., Bonar, M., Robitaille, A. L., Hart, C., Zabihi-Seissan, S., \& Wal, E. Vander. (2020). The ecology of individual differences empirically applied to spaceuse and movement tactics. The American Naturalist. The American Naturalist, (4), 1-4. doi: $10.1086 / 708721$ 
1171 Webber, Q. M. R., \& Vander Wal, E. (2018). An evolutionary framework outlining the integration of individual social and spatial ecology. Journal of Animal Ecology, 87(1), 113127. doi: $10.1111 / 1365-2656.12773$

Webber, Q. M. R., \& Vander Wal, E. (2019). Trends and perspectives on the use of animal social network analysis in behavioural ecology: a bibliometric approach. Animal Behaviour, 149, 77-87. doi: 10.1016/j.anbehav.2019.01.010

Weinstein, S. B., Buck, J. C., \& Young, H. S. (2018). A landscape of disgust. Science, 359(6381), 1213-1215.

White, L. A., Forester, J. D., \& Craft, M. E. (2017). Using contact networks to explore mechanisms of parasite transmission in wildlife. Biological Reviews, 92(1), 389-409. doi: $10.1111 /$ brv.12236

White, L. A., Forester, J. D., \& Craft, M. E. (2018). Dynamic, spatial models of parasite transmission in wildlife: Their structure, applications and remaining challenges. Journal of Animal Ecology, 87(3), 559-580. doi: 10.1111/1365-2656.12761

Whitehead, H. (2008). Analyzing animal societies : quantitative methods for vertebrate social analysis. University of Chicago Press.

Whitehead, H., \& James, R. (2015). Generalized affiliation indices extract affiliations from social network data. Methods in Ecology and Evolution, 6(7), 836-844. doi: 10.1111/2041210X.12383

Wilson-Aggarwal, J. K., Ozella, L., Tizzoni, M., Cattuto, C., Swan, G. J. F., Moundai, T., ... McDonald, R. A. (2019). High-resolution contact networks of free-ranging domestic dogs Canis familiaris and implications for transmission of infection. PLOS Neglected Tropical Diseases, 13(7), e0007565. doi: 10.1371/journal.pntd.0007565

Wood, M. J., Cosgrove, C. L., Wilkin, T. A., Knowles, S. C. L., Day, K. P., \& Sheldon, B. C. (2007). Within-population variation in prevalence and lineage distribution of avian malaria in blue tits, <i>Cyanistes caeruleus </ i>. Molecular Ecology, 16(15), 3263-3273. doi: 10.1111/j.1365-294X.2007.03362.x

Wood, S. N. (2011). Fast stable restricted maximum likelihood and marginal likelihood estimation of semiparametric generalized linear models. Journal of the Royal Statistical Society: Series B (Statistical Methodology), 73(1), 3-36. doi: 10.1111/j.14679868.2010.00749.x 
1202 Woodroffe, R., Donnelly, C. A., Ham, C., Jackson, S. Y. B., \& Moyes, K. (2016). Badgers prefer 1203 cattle pasture but avoid cattle : implications for bovine tuberculosis control. Ecology Letters, $1204 \quad 19,1201-1208$. doi: 10.1111/ele.12654

1205 